\title{
INFLUENCIA DE LA TEMPERATURA Y HUMEDAD AMBIENTAL DEL VERANO E INVIERNO SOBRE PARÁMETROS PRODUCTIVOS DE POLLOS DE CARNE CRIADOS EN LA CIUDAD DE LIMA
}

\author{
Effect of Summer and Winter Air Temperature and Humidity on Productive \\ Parameters of B roiler Chickens Raised in the City of Lima
}

Carlos Tolentino M. ${ }^{1}$, Eliana Icochea D. ${ }^{1,2}$, Pablo Reyna S. ${ }^{1}$ y Ricardo Valdivia R. ${ }^{3}$

\section{ResUMen}

\begin{abstract}
Se evaluó la influencia de la temperatura y humedad medioambiental sobre los parámetros productivos de pollos de carne de la línea Cobb-Vantress 500 criados por 45 días bajo el sistema de galpón abierto durante las estaciones de invierno y verano del 2004. Se utilizaron 720 pollos (360 aves por estación, empleándose 5 repeticiones con 34 machos y 38 hembras por grupo). Se evaluó el peso corporal, ganancia de peso, consumo de alimento, índice de conversión alimenticia, mortalidad,uniformidad de peso corporale índice de eficiencia productivo. Hubo diferencias significativas $(\mathrm{p}<0.05)$ entre las campañas de verano e invierno con relación al peso y ganancia de peso semanal. La mayor temperatura en el verano afectó el consumo de alimento, la ganancia de peso y el índice de eficiencia productiva, pero no el índice de conversión alimenticia. La crianza de pollos de carne, tanto machos como hembras, durante la estación de verano bajo sistemas de galpón abierto ocasionó una merma de 200 y 100 g de peso corporal, respectivamente, en comparación con la crianza en invierno.
\end{abstract}

Palabras clave: medio ambiente, estaciones, parámetros productivos

\section{Abstract}

This study was carried out to evaluate the influence of environmental temperature and humidity on productive performance of Cobb-Vantress 500 broiler chickens reared for 45 days under an open broiler house during the winter and summer seasons (2004). The trial comprised 720 1-day-old chickens (360 chickens per season using 5 repetitions of 34 males and 38 females each). Body weight, body weight gain, feed consumption, feed conversion index, mortality, body size uniformity, and productive efficiency index were evaluated. Weekly body weight and body weight gain were statistically different between birds reared in winter and summer $(\mathrm{p}<0.05)$. The higher temperature during summer affected feed consumption, body weight and productive efficiency index but did

\footnotetext{
${ }^{1}$ Laboratorio de Patología Aviar, Facultad de Medicina Veterinaria, Universidad Nacional Mayor de San Marcos, Lima

${ }^{2}$ E-mail: eliana.icochea@gmail.com

${ }^{3}$ Profesor Emérito, UNMSM
} 
not affect feed conversion index. The rearing of broiler chickens, both males and females, during the summer season as compared to winter season resulted in 200 and $100 \mathrm{~g}$ less per bird of body weight respectively.

Key words: environment, season, productive performance

\section{INTRODUCCIÓN}

La avicultura es las rama pecuaria del Perú con mayor crecimiento de los últimos años (Barbieri, 2001); sin embargo, el avance genético en el país no ha ido paralelo al desarrollo tecnológico de infraestructura para la crianza de aves como sucede en otras partes del mundo, manteniéndose el sistema de crianza empleado a galpón abierto, tal y como era en los inicios de la avicultura (Icochea, 1994).

Los parámetros productivos del pollo de carne difieren según la época del año en que son criados debido a la influencia de factores medio ambientales como temperatura y humedad. En estaciones con temperaturas elevadas, los parámetros productivos se afectan debido al estrés térmico que sufren las aves (Arce et al., 1992), ya que son muy sensibles a cambios del medio ambiente (North y Bell, 1990). Es necesario que el pollo reciba calor en época fría, refrescarlo en los días calurosos y brindarle una adecuada ventilación para reducir la humedad y mejorar la eliminación de gases nocivos que se producencentrodel galpón (Barragán, 2004).

Las aves son capaces de mantener la temperatura interna de sus órganos; sin embargo, este mecanismo de homeostasis solo es eficiente cuando la temperatura ambiental se encuentra dentro de ciertos límites (Cunningham, 1999). Cambios drásticos de temperatura y humedad, que no son adecuadamente controlados por el avicultor, afectan severamente el rendimiento productivo del pollo de carne, ocasionando grandes pérdidas económicas a los avicultores (Dale, 2002). En base a esto, se planteó el presente estudio a fin de comparar los parámetros productivos de pollos de carne criados bajo sistema de galpón abierto durante las estaciones de verano e invierno.

\section{Materiales y Métodos}

El estudio comprendió dos campañas consecutivas de crianza realizadas en el 2004 en el galpón de producción avícola de la Facultad de Medicina Veterinaria de la Universidad Nacional Mayor de San Marcos, LimaPerú.

El número mínimo de aves requerido para el estudio se calculó mediante la fórmula de diferencia de medias, indicando un tamaño muestral de, al menos, 96 aves por estación climática; sin embargo, por razones prácticas, se utilizaron 360 animales por estación. Las aves fueron pollos BB de carne de la línea Cobb-Vantress 500 de un día de edad. Se evaluó la campaña de verano (febrero a marzo) y la de invierno (junio a julio), empleándose 5 repeticiones con 34 machos y 38 hembras por grupo.

La crianza se realizó a galpón abierto, separándose las aves por sexo. El agua y alimento se administró ad libitum y se usaron campanas a gas como fuente de calor con temperaturas de $32^{\circ} \mathrm{C}$ en invierno y $30^{\circ} \mathrm{C}$ en verano durante la primera semana, reduciendo $3{ }^{\circ} \mathrm{C}$ por semana hasta la cuarta semana en invierno y hasta la tercera en verano. Se usó elel mismo tipo de alimento para machos y hembras durante todo el estudio, verificándose diariamente el manejo y el estado sanitario de las aves, así como la temperatura y humedad relativa medioambiental. 
La temperatura se midió con un termómetro digital ubicado fuera del área de las campanas, al centro del galpón, a 3-4 metros de la pared, y a $5 \mathrm{~cm}$ por encima de la cama (Dávila, 1994), registrándose los valores máximo y mínimo.

El peso corporal de cada ave y el consumo de alimento (ofrecido menos el sobrante) se registró semanalmente usando una balanza digital de $15 \mathrm{~kg}$ de capacidad con una precisión de $1 \mathrm{~g}$. El Índice de Conversión Alimenticia (ICA), parámetro que se define como la cantidad de alimento requerido para producir un kilogramo de peso vivo de pollo, se calculó semanalmente y a los 45 días de edad, en base a la siguiente fórmula: ICA = Alimento consumido (kg) / Peso vivo corporal $(\mathrm{kg})$.

Así mismo, se calculó el Índice Productivo Europeo (IPE), el cual se utiliza para evaluar el rendimiento integral de una parvada de pollos de engorde, ya que toma en consideración la mortalidad, el peso vivo promedio, la edad y la conversión alimenticia (Ivore, 1985). Se calculó con la siguiente fórmula: $\mathrm{IPE}=($ Supervivencia $)($ Peso promedio $) \times 100$ / (Días de edad) (ICA)

La Uniformidad (U) de tamaño del lote se calculó de la siguiente forma: $\mathrm{U}=$ Número de aves (Peso promedio $\pm 10 \%$ ) / 100 .

Las diferencias estadísticas entre lotes por efecto de la estación climática se analizaron a través de un análisis de varianza con arreglo factorial y la prueba de comparación múltiple de Duncan.

\section{Resultados}

Los promedios de la temperatura y humedad relativa se observan en la Fig. 1. El peso corporal de los pollos de carne durante los 45 días del estudio se encontró significativamente influenciado $(\mathrm{p}<0.05)$ por la estación climática de crianza, tanto en ma- chos como en hembras (Cuadro 1). El peso promedio de ambos sexos a los 45 días fue de $2.961 \mathrm{~kg}$ en el invierno y $2.809 \mathrm{~kg}$ en el verano $(\mathrm{p}<0.05)$.

El consumo total de alimento y el ICA fue significativamente afectado $(\mathrm{p}<0.05)$ por el efecto de la estación climática. En forma similar, los lotes de machos y hembras fueron más uniformes durante la campaña de invierno en comparación con la de verano $(\mathrm{p}<0.05)$ (Cuadro 2). El IPE, para la población global de machos y hembras, fue de 348.43 para la campaña de invierno y de 320.79 para la campaña de verano $(\mathrm{p}<0.05)$.

\section{Discusión}

Los pollos, según su edad, requieren de una temperatura y humedad relativa tal, que se mantenga dentro de ciertos rangos para que no afecte su eficiencia productiva, especialmente dentro de las tres primeras semanas de vida (Dávila, 1994). El pollo necesita un promedio de $30^{\circ} \mathrm{C}$ durante la primera semana de vida (Barragán, 2004; Hulzebosch, 2004), y en el presente estudio, en el invierno y pese a la calefacción, se registró un promedio de $27{ }^{\circ} \mathrm{C}$ de temperatura mínima, lo cual incrementó la sensibilidad térmica al frío. Esto puede haber motivado que el peso corporal de los machos en la primera semana fuera de $157 \mathrm{~g}$ en la campaña de invierno versus $177 \mathrm{~g}$ en la de verano.

No obstante esta desventaja inicial, los pollos machos criados en invierno llegaron a pesar $200 \mathrm{~g}$ más que aquellos criados en verano a los 45 días de iniciada la campaña. Esto, posiblemente, porque las aves necesitan una temperatura más baja a partir de la cuarta semana de edad, y en el estudio se llegaron a registrar temperaturas de 32 a 34 ${ }^{\circ} \mathrm{C}$ en la campaña de verano, cuando las aves necesitan alrededor de $24^{\circ} \mathrm{C}$ (Kwakernaak, 2000), afectando negativamente el consumo de alimento. Un efecto similar se pudo observar en las hembras aunque con menor 

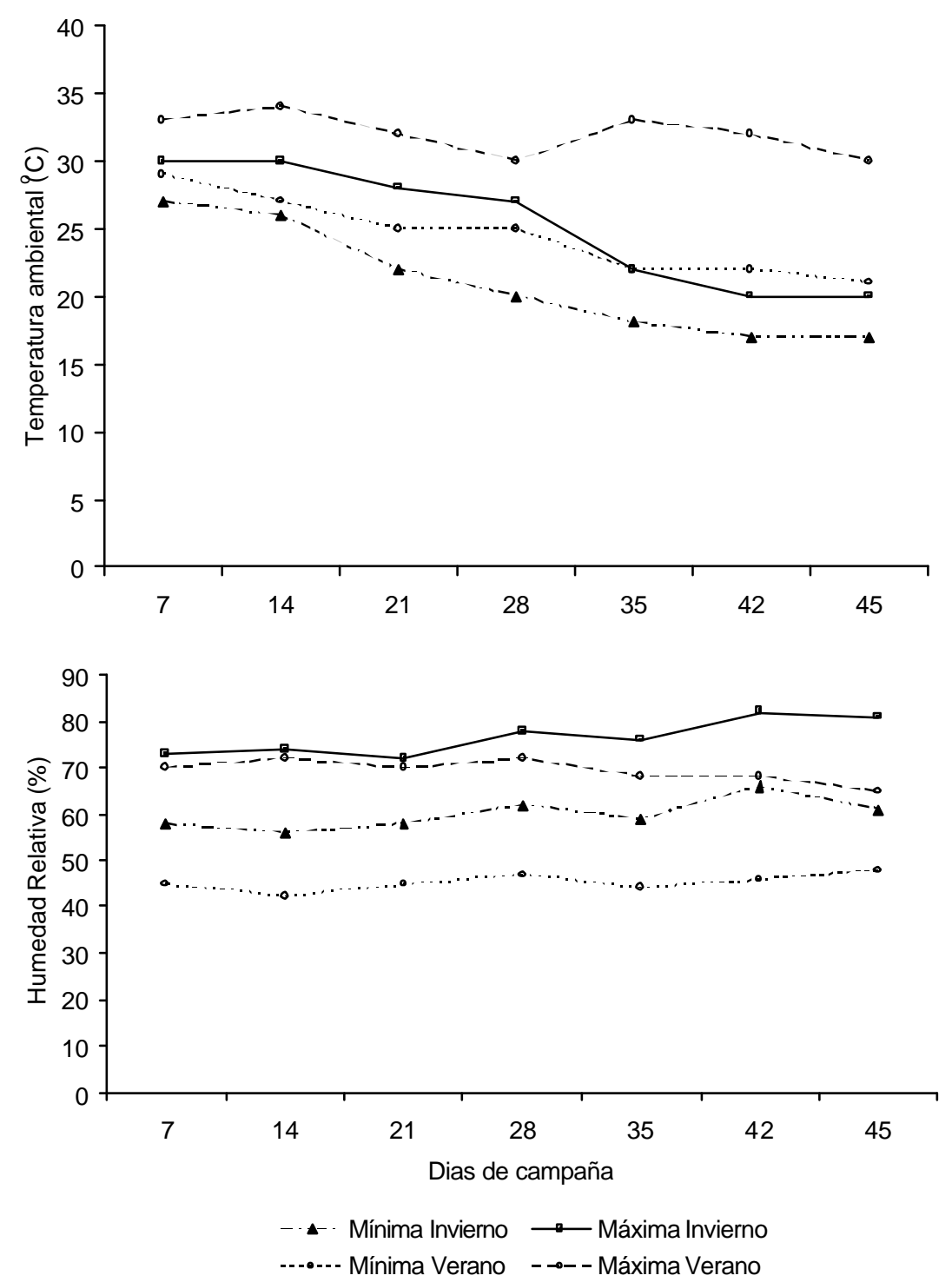

Figura 1. Temperatura $\left({ }^{\circ} \mathrm{C}\right)$ y humedad relativa (\%) mínima y máxima del ambiente del galpón de crianza de pollos BB de carne de la línea Cobb-Vantress 500 durante una campaña de verano (febrero a marzo) y otra de invierno (junio a julio) en el 2004

intensidad, dado que los machos, por su mayor peso corporal, son más sensibles a los cambios ambientales. El detrimento en la eficiencia productiva causada por las temperaturas elevadas del verano han sido ampliamente documentadas (Marrufo et al., 1999; Barragán, 2004).

Las diferencias en peso corporal y ganancia de peso guardan estrecha relación con el menor consumo de alimento de las aves criadas durante la estación de verano. Por ejemplo, en el presente estudio, las aves criadas en verano consumieron $17 \%$ menos de alimento durante la última semana de la campaña que las aves criadas en el invierno en el mismo periodo. Resultados similares han sido reportados por Qureshi, 2002 y Yalcin et al., 1997, citado por Ploog, 1998.

El menor ICA observado a los 45 días de edad en las aves criadas durante la estación de verano con relación a las aves criadas en invierno fue debido al menor consumo 
Cuadro 1. Peso corporal promedio $(\mathrm{kg})$ en pollos BB de carne de la línea Cobb-Vantress 500 a diferentes edades que fueron criados en un galpón experimental de la Universidad Nacional Mayor de San Marcos (2004)

\begin{tabular}{ccccc}
\hline \multirow{2}{*}{$\begin{array}{c}\text { Edad } \\
\text { días })\end{array}$} & \multicolumn{2}{c}{ Machos } & \multicolumn{2}{c}{ Hembras } \\
\cline { 2 - 5 } & Invierno & Verano & Invierno & Verano \\
\hline 1 & $0.045^{\mathrm{a}}$ & $0.046^{\mathrm{a}}$ & $0.045^{\mathrm{a}}$ & $0.046^{\mathrm{a}}$ \\
7 & $0.157^{\mathrm{a}}$ & $0.179^{\mathrm{b}}$ & $0.155^{\mathrm{a}}$ & $0.169^{\mathrm{b}}$ \\
14 & $0.422^{\mathrm{a}}$ & $0.478^{\mathrm{b}}$ & $0.403^{\mathrm{a}}$ & $0.428^{\mathrm{b}}$ \\
21 & $0.888^{\mathrm{a}}$ & $0.961^{\mathrm{b}}$ & $0.832^{\mathrm{a}}$ & $0.842^{\mathrm{a}}$ \\
28 & $1.531^{\mathrm{a}}$ & $1.534^{\mathrm{a}}$ & $1.401^{\mathrm{a}}$ & $1.332^{\mathrm{b}}$ \\
35 & $2.242^{\mathrm{a}}$ & $2.277^{\mathrm{a}}$ & $1.934^{\mathrm{a}}$ & $1.884^{\mathrm{b}}$ \\
42 & $2.989^{\mathrm{a}}$ & $2.934^{\mathrm{a}}$ & $2.442^{\mathrm{a}}$ & $2.440^{\mathrm{a}}$ \\
45 & $3.260^{\mathrm{a}}$ & $3.060^{\mathrm{b}}$ & $2.661^{\mathrm{a}}$ & $2.557^{\mathrm{b}}$ \\
\hline
\end{tabular}

a,b Promedios dentro de sexos y de una misma fila con letras diferentes muestran diferencia estadística $(p<0.05)$

Cuadro 2. Consumo de alimento $(\mathrm{kg})$ e Índice de Conversión Alimenticia hasta los 45 días de edad en pollos BB de carne de la línea Cobb-Vantress 500 que fueron criados en un galpón experimental de la Universidad Nacional Mayor de San Marcos (2004)

\begin{tabular}{lcccc}
\hline & \multicolumn{2}{c}{ Machos } & \multicolumn{2}{c}{ Hembras } \\
\hline & Invierno & Verano & Invierno & Verano \\
\hline Consumo de alimento (kg por pollo) & 5.815 & 5.404 & 5.046 & 4.755 \\
$\begin{array}{l}\text { Índice de Conversión Alimenticia } \\
\text { (ICA) }\end{array}$ & $1.784^{\mathrm{a}}$ & $1.766^{\mathrm{b}}$ & $1.896^{\mathrm{a}}$ & $1.859^{\mathrm{b}}$ \\
Mortalidad (\%) & 3.5 & 7.5 & $1-6$ & 6.1 \\
Uniformidad (\%) & 83.5 & 77.4 & 80.4 & 71.6 \\
Índice Productivo Europeo (IPE) & 391.74 & 356.17 & 306.95 & 286.98 \\
\hline $\begin{array}{l}\text { a,b Promedios dentro de la misma fila y por sexos con letras diferentes son estadísticamente diferentes } \\
(\mathrm{p}<0.05)\end{array}$
\end{tabular}

de alimento por los efectos de la alta temperatura ambiental. En las hembras se observó un efecto similar a los machos ya que el ICA fue mayor al final de la estación de invierno. Se sabe que en estas condiciones, los anima- les solo buscan la forma de eliminar el exceso de calor y dejan de comer, jadeando constantemente para disipar el calor y sobrevivir a los efectos del estrés térmico (Kwakernaak, 2000; Van der Sluis, 2004). 
El IPE es una herramienta de gran utilidad para comparar la eficiencia productiva de lotes de pollos. En el presente estudio se encontró una mejor IPE en las aves criadas durante la estación de invierno (348.43) que en aquellas criadas durante la estación de verano (320.79), indicando que la falta de galpones climatizados impide controlar las variaciones térmicas que se ajusten a las exigencias de la capacidad genética del pollo actual, afectando el rendimiento económico de las aves (Dávila, 1994). En este estudio, aun cuando se trabajó con una pequeña población de aves y en ausencia de otros factores o condiciones de estrés, fueron notorios los efectos adversos del calor sobre la productividad de las aves. Estos efectos serían de mayor impacto en aves criadas bajo condiciones de campo, afectando no solo la productividad, sino también incrementando la susceptibilidad de las aves a sufrir de infecciones de diversa índole.

\section{Conclusiones}

Los pollos de carne criados durante la estación de invierno demostraron un mayor peso corporal, ganancia de peso, consumo de alimento, uniformidad de pesos e índice de eficiencia productiva, así como una menor mortalidad, en comparación con pollos criados en el verano.

\section{Literatura Citada}

1. Arce A, Ruiz B, Navarro H. 1992. Manual de estrés calórico en aves. Rev Asoc Amer Soya, México 1: 7-32.

2. Barbieri B. 2001. Reseña histórica de la industria avícola peruana. Rev Cienc Vet, Perú 17: 5-8.

3. Barragán J. 2004. Estrés térmico en aves. Mundo Avícola y Porcino, Perú 5: 10-13.

4. Cunningham J. 1999. Fisiología veterinaria. $2^{\mathrm{a}}$ ed. México: McGraw-Hill Interamericana. $763 \mathrm{p}$.
5. Dale N. 2002. Mejorando la competitividad de la producción avícola por medio de los programas de nutrición y alimentación. Mundo Avícola y Porcino, Perú 14: 22-23.

6. Dávila E. 1994. Eventos claves en la crianza de pollos parrilleros. I Curso Nacional de Producción y Sanidad Avícola para Técnicos. Lima: FMVUNMSM. p 12-19.

7. Hulzebosch J. 2004. What affects the climate in poultry houses? World Poultry 20: $36-38$.

8. Icochea E. 1994. Enfermedades más comunes de las aves en granja. I Curso Nacional de Producción y Sanidad Avícola para Técnicos. Lima: FMVUNMSM. p 43-46.

9. Kwakernaak C. 2000. Day and night temperatures and ascites in broilers. World Poultry 16: 20-21.

10. Marrufo D, Quintana J, Castañeda P. 1999. Efecto de la ventilación por presión positiva sobre los parámetros productivos de pollos de engorda, durante siete semanas en casetas de ambiente natural. Vet Méx 3: 99-102.

11. North M, Bell D. 1990. Commercial chicken production manual. $4^{\text {th }}$ ed. London: Chapman \& Hall. Cork.

12. Ploog H. 1998. El impacto del estrés calórico sobre la producción avícola. Mundo Avícola y Porcino, Perú 25: 14-17.

13. Qureshi A. 2002. Galpones de extremos abiertos para ponedoras en zonas de clima cálido. Avicultura Profesional, Perú 9: 24-26.

14. Spiegel M. 1998. Estadística. $2^{\mathrm{a}}$ ed. México: McGraw-Hill Interamericana.

15. Van der Sluis W. 2004. Floor temperature affects broilers performance. World Poultry 20: 17-19.

16. Yvore P. 1985. The use of shuttle programs for the control of coccidiosisin broilers. Proc Georgia Coccidiosis Conf. Georgia, USA. 